\title{
Comments on some recent generalization of the Banach contraction principle
}

Tomonari Suzuki*

\section{"Correspondence:}

suzuki-t@mns.kyutech.ac.jp

Department of Basic Sciences,

Faculty of Engineering, Kyushu

Institute of Technology, Tobata,

Kitakyushu 804-8550, Japan

\begin{abstract}
We study Browder and CJM contractions of integral type. As a result, we give an alternative proof of some recent generalization of the Banach contraction principle by Jleli and Samet.

MSC: Primary 47H09; secondary $54 \mathrm{H} 25$

Keywords: the Banach contraction principle; Browder contraction; CJM contraction; contraction of integral type; fixed point
\end{abstract}

\section{Introduction}

We have introduced many types of contractions. We give the definitions of three of them. Define $\Phi$ as follows: $\varphi \in \Phi$ iff $\varphi$ is a nondecreasing, right continuous function from $[0, \infty)$ into itself satisfying $\varphi(t)<t$ for any $t>0$. It is obvious that $\varphi(0)=0$ holds.

Definition 1 Let $T$ be a mapping on a metric space $(X, d)$.

- $T$ is said to be a (usual) contraction ( $C$, for short) $[1,2]$ if there exists $r \in[0,1)$ such that $d(T x, T y) \leq r d(x, y)$ for any $x, y \in X$.

- $T$ is said to be a Browder contraction (BroC, for short) [3] if there exists $\varphi \in \Phi$ such that $d(T x, T y) \leq \varphi \circ d(x, y)$ for any $x, y \in X$.

- $T$ is said to be a CJM contraction (CJMC, for short) [4-6] if the following hold:

(j) For every $\varepsilon>0$, there exists $\delta>0$ such that $d(x, y)<\varepsilon+\delta$ implies $d(T x, T y) \leq \varepsilon$.

(jj) $x \neq y$ implies $d(T x, T y)<d(x, y)$.

We know the following implications:

$$
\mathrm{C} \Longrightarrow \mathrm{BroC} \Longrightarrow \mathrm{CJMC} \text {. }
$$

There are some conditions equivalent to BroC; see [7]. Lemma 5 in [8] gives seven equivalent conditions connected with BroC. See [9] and the references therein for further information as regards contractions.

Branciari [10] introduced contractions of integral type as follows: A mapping $T$ on a metric space $(X, d)$ is a Branciari contraction if there exist $r \in[0,1)$ and a locally integrable

(c) 2016 Suzuki. This article is distributed under the terms of the Creative Commons Attribution 4.0 International License (http://creativecommons.org/licenses/by/4.0/), which permits unrestricted use, distribution, and reproduction in any medium, provided you give appropriate credit to the original author(s) and the source, provide a link to the Creative Commons license, and indicate if changes were made. 
function $f$ from $[0, \infty)$ into itself such that

$$
\int_{0}^{s} f(t) d t>0 \quad \text { and } \quad \int_{0}^{d(T x, T y)} f(t) d t \leq r \int_{0}^{d(x, y)} f(t) d t
$$

for all $s>0$ and $x, y \in X$.

We have studied contractions of integral type in $[11,12]$. Very recently, Jleli and Samet [13] introduced the following new type of contractions.

Theorem 2 (Jleli and Samet [13]) Let $(X, d)$ be a complete generalized metric space and let $\theta$ be a function from $(0, \infty)$ into $(1, \infty)$ satisfying the following:

$(\theta 1) \theta$ is nondecreasing.

$(\theta 2)$ For any sequence $\left\{t_{n}\right\}$ in $(0, \infty), \lim _{n} \theta\left(t_{n}\right)=1$ iff $\lim _{n} t_{n}=0$.

(日3) There exist $r \in(0,1)$ and $\ell \in(0, \infty]$ such that $\lim _{t \rightarrow+0}(\theta(t)-1) / t^{r}=\ell$.

Let $T$ be a mapping on $X$. Assume that there exists $k \in(0,1)$ such that

$$
T x \neq T y \quad \text { implies } \quad \theta \circ d(T x, T y) \leq(\theta \circ d(x, y))^{k}
$$

for any $x, y \in X$. Then $T$ has a unique fixed point.

\section{Remark}

- Considering the domain and range of $\theta$ and ( $\theta 1)$, it is obvious that $(\theta 2)$ is equivalent to $\inf \{\theta(t): t \in(0, \infty)\}=1$.

- The underlying space of Theorem 2 is a generalized metric space. This interesting concept was introduced by Branciari [14]. See also [15-18] and others. However, we omit the statement of the definition of a generalized metric space because it is not essential in this paper.

In this paper, motivated by Theorem 2, we deepen the study of contractions of integral type. We also give an alternative proof of Theorem 2 .

\section{Preliminaries}

Throughout this paper we denote by $\mathbb{N}$ the set of all positive integers and by $\mathbb{R}$ the set of all real numbers. For a function $f$, we denote by $\operatorname{Dom}(f)$ the domain of $f$.

Let $f$ be a function from a subset of $\mathbb{R}$ into $\mathbb{R}$. Then $f$ is said to satisfy $(\mathrm{U})_{f}$ if the following holds:

$(\mathrm{U})_{f}$ For any $t \in \operatorname{Dom}(f)$, there exist $\delta>0$ and $\varepsilon>0$ such that $f(s) \leq t-\varepsilon$ holds for any $s \in(t-\delta, t+\delta) \cap \operatorname{Dom}(f)$.

We list some further notation in order to simplify the statement of the results of this paper:

(A1) Let $Y$ be an arbitrary set and let $h$ be a function from $Y$ into $[0, \infty)$. Let $S$ be a mapping on $Y$ satisfying that $h(x)=0$ implies $h(S x)=0$ for any $x \in Y$.

(A2) Let $\theta$ be a function from $(0, \infty)$ into $\mathbb{R}$. Put $\Theta=\theta((0, \infty))$ and

$$
\Theta_{\leq}=\bigcup\{[t, \infty): t \in \Theta\} .
$$


The purpose of this study is to obtain the mathematical structure of contractions mentioned in Section 1. So, we simplify our setting as follows:

Definition 3 Assume (A1).

- $S$ is said to be a Browder contraction if there exists $\varphi \in \Phi$ such that

$$
h(S x) \leq \varphi \circ h(x)
$$

for any $x \in Y$.

- $S$ is said to be a CJM contraction if the following hold:

(j) For any $\varepsilon>0$, there exists $\delta>0$ such that $h(x)<\varepsilon+\delta$ implies $h(S x) \leq \varepsilon$.

(j) $h(x)>0$ implies $h(S x)<h(x)$.

Remark Let $(X, d)$ and $T$ be as in Definition 1. Put $Y=X \times X$ and define $h$ and $S$ by $h((x, y))=d(x, y)$ and $S(x, y)=(T x, T y)$. Then the concept of Browder contraction in Definition 3 becomes that in Definition 1 and the concept of CJM contraction in Definition 3 becomes that in Definition 1.

We give some lemmas concerning $(\mathrm{U})_{f}$.

Lemma 4 Let $f$ be a function from a subset of $\mathbb{R}$ into $\mathbb{R}$. Then $f$ satisfies $(\mathrm{U})_{f}$ iff

$$
\lim \sup [f(u): u \rightarrow t, u \in \operatorname{Dom}(f)]<t
$$

holds for any $t \in \operatorname{Dom}(f)$.

Remark We define $\lim \sup [f(u): u \rightarrow t, u \in \operatorname{Dom}(f)]=\gamma$ iff the following hold:

- There exists a sequence $\left\{u_{n}\right\}$ in $\operatorname{Dom}(f)$ such that $\left\{u_{n}\right\}$ converges to $t$ and $\left\{f\left(u_{n}\right)\right\}$ converges to $\gamma$.

- $\lim \sup _{n} f\left(u_{n}\right) \leq \gamma$ holds for any sequence $\left\{u_{n}\right\}$ in $\operatorname{Dom}(f)$ converging to $t$.

Note that we do not exclude the case of $u_{n}=t$.

Proof of Lemma 4 Obvious.

Lemma 5 Let $f$ be an upper semicontinuous function from a subset of $\mathbb{R}$ into $\mathbb{R}$ such that $f(t)<t$ for any $t \in \operatorname{Dom}(f)$. Then $f$ satisfies $(\mathrm{U})_{f}$.

Proof Obvious.

Lemma 6 Let $\psi$ be a function from a subset $D$ of $\mathbb{R}$ into $\mathbb{R}$ satisfying $(\mathrm{U})_{\psi}$. Let $M$ be a real number with $M>1$. Define a function $\varphi$ from $D$ into $\mathbb{R}$ by

$$
\begin{aligned}
\varphi(t)= & \frac{t}{2}+\frac{1}{2} \max \{\sup \{\psi(u): u \in D, u \leq t\}, \\
& \sup \{\psi(u)+M(t-u): u \in D, t \leq u\}\}
\end{aligned}
$$

for $t \in D$. Then the following hold: 
(i) $\varphi$ satisfies $(\mathrm{U})_{\varphi}$.

(ii) $\varphi$ is strictly increasing and Lipschitz continuous.

(iii) $\psi(t)<\varphi(t)$ holds for any $t \in D$.

Proof In this proof, we always assume $s, t, u \in D$. Define a function $\omega$ from $D$ into $\mathbb{R}$ by

$$
\omega(t)=\max \{\sup \{\psi(u): u \leq t\}, \sup \{\psi(u)+M(t-u): t \leq u\}\} .
$$

For each $u$, we define a function $\psi_{u}$ from $D$ into $\mathbb{R}$ by

$$
\psi_{u}(t)= \begin{cases}\psi(u)+M(t-u) & \text { if } t \leq u \\ \psi(u) & \text { if } t \geq u\end{cases}
$$

Then from the definition of $\omega$, we note

$$
\omega(t)=\sup \left\{\psi_{u}(t): u\right\}
$$

So it is obvious that $\psi(t) \leq \omega(t)$. We can easily check that $\psi_{u}$ is nondecreasing and $\left|\psi_{u}(s)-\psi_{u}(t)\right| \leq M|s-t|$ holds. Hence $\omega$ is nondecreasing and

$$
|\omega(s)-\omega(t)| \leq M|s-t|
$$

holds. Hence $\omega$ is continuous. Fix $t$; and choose $\delta$ and $\varepsilon$ as in the definition of $(\mathrm{U})_{f}$. We have

$$
\begin{aligned}
\omega(t)= & \max \left\{\sup _{u \leq t-\delta} \psi_{u}(t), \sup _{t-\delta<u \leq t} \psi_{u}(t), \sup _{t \leq u<t+\delta} \psi_{u}(t), \sup _{t+\delta \leq u} \psi_{u}(t)\right\} \\
= & \max \left\{\sup _{u \leq t-\delta} \psi(u), \sup _{t-\delta<u \leq t} \psi(u),\right. \\
& \left.\sup _{t \leq u<t+\delta}(\psi(u)+M(t-u)), \sup _{t+\delta \leq u}(\psi(u)+M(t-u))\right\} \\
\leq & \max \left\{\sup _{u \leq t-\delta} u, \sup _{t-\delta<u<t+\delta} \psi(u), \sup _{t+\delta \leq u}(u+M(t-u))\right\} \\
\leq & \max \{t-\delta, t-\varepsilon, t+\delta-M \delta\} \\
< & t .
\end{aligned}
$$

Hence by Lemma $5, \omega$ satisfies $(\mathrm{U})_{\omega}$. It is obvious that

$$
|\varphi(s)-\varphi(t)| \leq \frac{M+1}{2}|s-t| \quad \text { and } \quad \psi(t) \leq \omega(t)<\varphi(t)<t
$$

hold. We can easily prove the remainders.

Remark We use the method in the proof of Proposition 1 in [19]. Note that the domain of $\varphi$ is $D$. We cannot extend the domain of $\varphi$ to $\bigcup\{[t, \infty): t \in D\}$. See $\psi$ in Example 15 below. 


\section{Browder contraction}

In this section, we discuss Browder contractions.

Lemma 7 Assume (A1). Let $\psi$ be a function from $(0, \infty)$ into $[0, \infty)$ such that $(\mathrm{U})_{\psi}$ holds and

$$
h(S x) \leq \psi \circ h(x)
$$

for any $x \in Y$ with $h(x)>0$ and $h(S x)>0$. Then $S$ is a Browder contraction.

Proof By Lemma 6, there exists a nondecreasing continuous function $\eta$ from $(0, \infty)$ into $[0, \infty)$ such that $(\mathrm{U})_{\eta}$ is satisfied and $\psi(t) \leq \eta(t)$ holds for any $t \in(0, \infty)$. Define a function $\varphi$ from $[0, \infty)$ into itself by

$$
\varphi(t)= \begin{cases}\eta(t) & \text { if } t>0 \\ 0 & \text { if } t=0\end{cases}
$$

It is obvious that $\varphi$ is nondecreasing continuous function such that $\varphi(t)<t$ for any $t \in$ $(0, \infty)$. Thus, $\varphi \in \Phi$. Fix $x \in Y$. We consider the following two cases:

- $h(S x)=0$,

- $h(S x)>0$.

In the first case, $h(S x) \leq \varphi \circ h(x)$ obviously holds. In the second case, we note that $h(S x)>0$ implies $h(x)>0$. So $h(x)>0$ holds. We have

$$
h(S x) \leq \psi \circ h(x) \leq \eta \circ h(x)=\varphi \circ h(x) .
$$

Therefore $S$ is a Browder contraction.

Proposition 8 Assume (A1), (A2) and the following:

(i) $\theta$ is nondecreasing and continuous.

(ii) There exists a function $\psi$ from $\Theta$ into $\mathbb{R}$ satisfying $(\mathrm{U})_{\psi}$ and

$$
\theta \circ h(S x) \leq \psi \circ \theta \circ h(x)
$$

for any $x \in Y$ with $h(x)>0$ and $h(S x)>0$.

Then $S$ is a Browder contraction.

Proof Define a function $\theta_{+}^{-1}$ from $\mathbb{R}$ into $[0, \infty]$ by

$$
\theta_{+}^{-1}(\tau)= \begin{cases}\sup \{s \in(0, \infty): \theta(s) \leq \tau\} & \text { if }\{s \in(0, \infty): \theta(s) \leq \tau\} \neq \varnothing \\ 0 & \text { otherwise }\end{cases}
$$

Put $\varphi=\theta_{+}^{-1} \circ \psi \circ \theta$. We note

$$
\varphi(t)=\sup \{s \in(0, \infty): \theta(s) \leq \psi \circ \theta(t)\} \quad \text { provided } \varphi(t)>0 .
$$


Since $\psi \circ \theta(t)<\theta(t) \leq \theta(s)$ for any $t, s \in(0, \infty)$ with $t \leq s$, we have $\varphi(t) \leq t$ for any $t \in$ $(0, \infty)$. Thus, $\varphi$ is a function from $(0, \infty)$ into $[0, \infty)$. Arguing by contradiction, we assume that $(\mathrm{U})_{\varphi}$ does not hold. Then there exist $t \in(0, \infty)$ and a sequence $\left\{t_{n}\right\}$ in $(0, \infty)$ such that $\left\{t_{n}\right\}$ converges to $t$ and

$$
\varphi\left(t_{n}\right)>(1-1 / n) t
$$

holds for $n \in \mathbb{N}$. Since $\varphi\left(t_{n}\right)>0$,

$$
\sup \left\{s \in(0, \infty): \theta(s) \leq \psi \circ \theta\left(t_{n}\right)\right\}>(1-1 / n) t
$$

holds. Hence there exists a sequence $\left\{u_{n}\right\}$ in $(0, \infty)$ satisfying

$$
\theta\left(u_{n}\right) \leq \psi \circ \theta\left(t_{n}\right)<\theta\left(t_{n}\right) \text { and } u_{n}>(1-2 / n) t
$$

for $n \in \mathbb{N}$. Since $\theta$ is nondecreasing, $u_{n}<t_{n}$ holds for any $n \in \mathbb{N}$. Thus $\left\{u_{n}\right\}$ also converges to $t$. Hence

$$
\theta(t) \leq \limsup _{n \rightarrow \infty} \psi \circ \theta\left(t_{n}\right) \leq \lim \sup [\psi(\tau): \tau \rightarrow \theta(t), \tau \in \Theta]
$$

because $\theta$ is continuous. This contradicts $(U)_{\psi}$. Therefore $(U)_{\varphi}$ holds. For any $x \in Y$ with $h(x)>0$ and $h(S x)>0$, we have by $(1)$

$$
h(S x) \leq \sup \{s \in(0, \infty): \theta(s) \leq \psi \circ \theta \circ h(x)\}=\varphi \circ h(x) .
$$

Therefore by Lemma 7, $S$ is a Browder contraction.

By Lemma 5, we obtain the following.

Corollary 9 Assume (A1), (A2), (i) in Proposition 8 and the following:

(ii) There exists an upper semicontinuous function $\psi$ from $\Theta$ into $\mathbb{R}$ satisfying $\psi(t)<t$ for any $t \in \Theta$ and (1) for any $x \in Y$ with $h(x)>0$ and $h(S x)>0$.

Then $S$ is a Browder contraction.

The following examples tell that the continuity of $\theta$ in Proposition 8 is needed. In Example 10, $\theta$ is right continuous, however, it is not left continuous. On the other hand, in Example 11, $\theta$ is left continuous, however, it is not right continuous.

Example 10 (Example 2.3 in [12]) Define a complete metric space $(X, d)$ by

$$
X=[0,1] \cup[2, \infty) \quad \text { and } \quad d(x, y)= \begin{cases}\min \{x+y, 2\} & \text { if } x \neq y \\ 0 & \text { if } x=y\end{cases}
$$

Define a mapping $T$ on $X$ and functions $\theta$ and $\psi$ from $(0, \infty)$ into itself by

$$
T x=\left\{\begin{array}{ll}
0 & \text { if } x \leq 1, \\
1-1 / x & \text { if } x \geq 2,
\end{array} \quad \theta(t)= \begin{cases}1 & \text { if } t<2 \\
2 & \text { if } t \geq 2\end{cases}\right.
$$


and $\psi(t)=t / 2$. Put $Y=X \times X$ and define $h$ and $S$ by $h((x, y))=d(x, y)$ and $S(x, y)=(T x, T y)$ for $(x, y) \in Y$. Then all the assumptions of Proposition 8 except the left continuity of $\theta$ are satisfied. However, $T$ is not a Browder contraction.

Example 11 (Example 2.6 in [11]) Define a complete metric space $(X, d)$ by $X=[0, \infty)$ and $d(x, y)=x+y$ for $x, y \in X$ with $x \neq y$. Define a mapping $T$ on $X$ and functions $\theta$ and $\psi$ from $(0, \infty)$ into itself by

$$
T x=\left\{\begin{array}{ll}
0 & \text { if } x \leq 1, \\
1 & \text { if } x>1,
\end{array} \quad \theta(t)= \begin{cases}t & \text { if } t \leq 1, \\
1+t & \text { if } t>1\end{cases}\right.
$$

and $\psi(t)=t / 2$. Let $Y, h$, and $S$ be as in Example 10. Then all the assumptions of Proposition 8 except the right continuity of $\theta$ are satisfied. However, $T$ is not a Browder contraction.

\section{CJM contraction}

In this section, we discuss CJM contractions.

The following is a modification of Proposition 2.6 in [12].

Proposition 12 Assume (A1), (A2), and the following:

(i) $\theta$ is nondecreasing.

(ii) For any $\varepsilon \in \Theta_{\leq}$, there exists $\delta>0$ such that $h(x)>0, h(S x)>0$, and $\theta \circ h(x)<\varepsilon+\delta$ imply $\theta \circ h(S x) \leq \varepsilon$ for all $x \in Y$.

(iii) $h(x)>0$ and $h(S x)>0$ imply $\theta \circ h(S x)<\theta \circ h(x)$.

Then $S$ is a CJM contraction.

Proof In order to show (jj), we fix $x \in Y$ with $h(x)>0$. Arguing by contradiction, we assume $h(S x) \geq h(x)$. Then $h(S x)>0$ obviously holds. We have from (iii),

$$
\theta \circ h(S x)<\theta \circ h(x)
$$

which contradicts (i). Therefore we obtain $h(S x)<h(x)$. We have shown (jj). We shall prove (j). Fix $\varepsilon>0$ and put $\beta=\lim [\theta(t): t \rightarrow \varepsilon+0]$. We consider the following two cases:

- $\beta<\theta(\varepsilon+\gamma)$ holds for any $\gamma>0$.

- There exists $\delta_{2}>0$ such that $\beta=\theta\left(\varepsilon+\delta_{2}\right)$.

In the first case, since $\theta(\varepsilon) \leq \beta, \beta \in \Theta_{\leq}$holds. From (ii), there exists $\alpha>0$ such that

$$
h(x)>0, \quad h(S x)>0 \quad \text { and } \quad \theta \circ h(x)<\beta+\alpha \quad \text { imply } \quad \theta \circ h(S x) \leq \beta .
$$

We can choose $\delta_{1}>0$ satisfying $\theta\left(\varepsilon+\delta_{1}\right)<\beta+\alpha$. Fix $x \in Y$ with $h(x)<\varepsilon+\delta_{1}$. Arguing by contradiction, we assume $h(S x)>\varepsilon$. Then we have $h(S x)>0$ and hence $h(x)>0$. We have

$$
\theta \circ h(x) \leq \theta\left(\varepsilon+\delta_{1}\right)<\beta+\alpha
$$

and hence

$$
\beta<\theta \circ h(S x) \leq \beta,
$$


which is a contradiction. Therefore we obtain $h(S x) \leq \varepsilon$. In the second case, we also fix $x \in Y$ with $h(x)<\varepsilon+\delta_{2}$. Arguing by contradiction, we assume $h(S x)>\varepsilon$. Then we have $h(S x)>0$ and hence $h(x)>0$. By (iii), we have

$$
\beta \leq \theta \circ h(S x)<\theta \circ h(x) \leq \theta\left(\varepsilon+\delta_{2}\right)=\beta,
$$

which is a contradiction. Therefore we obtain $h(S x) \leq \varepsilon$. We have proven (j).

The proof of the following is obvious, however, we give a proof in order to show how differently $\Theta$ and $\Theta_{\leq}$work.

Corollary 13 Assume (A1), (A2), (i) in Proposition 12 and the following:

(ii) There exists a function $\psi$ from $\Theta_{\leq}$into $\mathbb{R}$ satisfying $(\mathrm{U})_{\psi}$ and (1) for any $x \in Y$ with $h(x)>0$ and $h(S x)>0$.

Then $S$ is a CJM contraction.

Proof We first note that from $(\mathrm{U})_{\psi}, \psi(t)<t$ for any $t \in \operatorname{Dom}(\psi)=\Theta_{\leq}$. For $x \in Y$ with $h(x)>0$ and $h(S x)>0$, we have

$$
\theta \circ h(S x) \leq \psi \circ \theta \circ h(x)<\theta \circ h(x)
$$

We have shown (iii) in Proposition 12. Let us prove (ii) in Proposition 12. Fix $\varepsilon \in \Theta_{\leq}$. Then from $(\mathrm{U})_{\psi}$, there exists $\delta>0$ such that $\psi(s)<\varepsilon$ holds for any $s \in[\varepsilon, \varepsilon+\delta)$. Fix $x \in Y$ with $h(x)>0, h(S x)>0$, and $\theta \circ h(x)<\varepsilon+\delta$. Then if $\theta \circ h(x)<\varepsilon$, then we have

$$
\theta \circ h(S x)<\theta \circ h(x)<\varepsilon .
$$

If $\theta \circ h(x) \geq \varepsilon$, then we have

$$
\theta \circ h(S x) \leq \psi \circ \theta \circ h(x)<\varepsilon
$$

Therefore by Proposition 12, $S$ is a CJM contraction.

By Lemma 5, we obtain the following.

Corollary 14 Assume (A1), (A2), (i) in Proposition 12 and the following:

(ii) There exists an upper semicontinuous function $\psi$ from $\Theta_{\leq}$into $\mathbb{R}$ satisfying $\psi(t)<t$ for any $t \in \Theta_{\leq}$and (1) for any $x \in Y$ with $h(x)>0$ and $h(S x)>0$.

Then $S$ is a CJM contraction.

There is a counterexample if we replace $\Theta_{\leq}$with $\Theta$ in Corollary 14 .

Example 15 Define a complete metric space $(X, d)$ by

$$
X=[1, \infty) \quad \text { and } \quad d(x, y)= \begin{cases}1+1 / x+1 / y & \text { if } x \neq y \\ 0 & \text { if } x=y\end{cases}
$$


Define a mapping $T$ on $X$ by $T x=2 x$ for $x \in X$. Define functions $\theta$ from $(0, \infty)$ into $\mathbb{R}$ and $\psi$ from $(0,1] \cup(2, \infty)$ into $\mathbb{R}$ by

$$
\theta(t)=\left\{\begin{array}{ll}
t & \text { if } t \leq 1, \\
1+t & \text { if } t>1,
\end{array} \text { and } \quad \psi(t)= \begin{cases}t / 2 & \text { if } t \leq 1 \\
1+t / 2 & \text { if } t>2\end{cases}\right.
$$

Let $Y, h$, and $S$ be as in Example 10. Then all the assumptions of Corollary 14 except $\operatorname{Dom}(\psi)=\Theta_{\leq}$are satisfied. However, $T$ is not a CJM contraction.

Remark We note that $\theta$ is left continuous, however, $\theta$ is not right continuous.

Proof For any $x, y \in X$ with $x \neq y$ and $T x \neq T y$, we have

$$
\psi \circ \theta \circ d(x, y)=\psi \circ \theta\left(1+\frac{1}{x}+\frac{1}{y}\right)=\psi\left(2+\frac{1}{x}+\frac{1}{y}\right)=2+\frac{1}{2 x}+\frac{1}{2 y}
$$

and

$$
\theta \circ d(T x, T y)=\theta \circ d(2 x, 2 y)=\theta\left(1+\frac{1}{2 x}+\frac{1}{2 y}\right)=2+\frac{1}{2 x}+\frac{1}{2 y} .
$$

Thus, (1) holds. Since $T$ has no fixed point, $T$ is not a CJM contraction.

We assume additionally that $\theta$ is right continuous. Then we can prove the following.

Proposition 16 Assume (A1), (A2), (iii) in Proposition 12 and the following:

(i) $\theta$ is nondecreasing and right continuous.

(ii) For any $\varepsilon \in \Theta$, there exists $\delta>0$ such that $h(x)>0, h(S x)>0$, and $\theta \circ h(x)<\varepsilon+\delta$ imply $\theta \circ h(S x) \leq \varepsilon$ for all $x \in Y$.

Then $S$ is a CJM contraction.

Proof We will show (ii) in Proposition 12. Fix $\varepsilon \in \Theta_{\leq} \backslash \Theta$. Then there exists $\delta>0$ such that

$$
[\varepsilon, \varepsilon+\delta] \subset \Theta_{\leq} \backslash \Theta
$$

If not so, then there exists a sequence $\left\{t_{n}\right\}$ in $(0, \infty)$ such that $\left\{\theta\left(t_{n}\right)\right\}$ is strictly decreasing and converges to $\varepsilon$. Since $\theta$ is nondecreasing, $\left\{t_{n}\right\}$ is strictly decreasing. Since $\left\{t_{n}\right\}$ is bounded from below, $\left\{t_{n}\right\}$ converges to some $t \in[0, \infty)$. Since $\varepsilon \in \Theta_{\leq}, t>0$ holds. Since $\theta$ is right continuous, we obtain $\theta(t)=\varepsilon$, which implies $\varepsilon \in \Theta$. This is a contradiction. Fix $x \in Y$ with $h(x)>0, h(S x)>0$, and $\theta \circ h(x)<\varepsilon+\delta$. Then we have $\theta \circ h(x)<\varepsilon$. Hence we obtain from (iii) (in Proposition 12)

$$
\theta \circ h(S x)<\theta \circ h(x)<\varepsilon
$$

So all the assumptions in Proposition 12 are satisfied. Therefore $S$ is a CJM contraction.

Corollary 17 Assume (A1), (A2), (i) in Proposition 16 and (ii) in Proposition 8. Then $S$ is a CJM contraction. 
Proof We can prove (iii) in Proposition 16 (in Proposition 12) as in the proof of Corollary 13. Also, we can prove (ii) in Proposition 16 as in the proof of Corollary 13. Therefore by Proposition 16, $S$ is a CJM contraction.

By Lemma 5, we also obtain the following.

Corollary 18 Assume (A1), (A2), (i) in Proposition 16 and (ii) in Corollary 9. Then S is a CJM contraction.

Finally, using Corollary 14, we give an alternative proof of Theorem 2.

Proof of Theorem 2 Let $Y, h$, and $S$ be as in Example 10. Define a function $\psi$ from $(1, \infty)$ into $(1, \infty)$ by $\psi(t)=t^{k}$. Since $\psi$ is continuous and $\psi(t)<t$ for any $t \in(1, \infty)$, all the assumption of Corollary 14 are satisfied. So by Corollary $14, S$ is a CJM contraction. By Theorem 13 in [16], $T$ has a unique fixed point $z$. Moreover, $\lim _{n} d\left(T^{n} x, z\right)=0$ for any $x \in X$.

\section{Remark}

(i) We do not need $(\theta 2)$ and $(\theta 3)$ in Theorem 2.

(ii) If we assume additionally that $\theta$ is continuous, then $T$ is a Browder contraction.

\section{Some comments}

As stated in Section 1, Lemma 5 in Jachymski [8] gives seven equivalent conditions connected with BroC. Finally, by Proposition 8 and Corollary 9 we can obtain the following, which is similar to Lemma 5 in [8].

Lemma 19 Let $D$ be a subset of $(0, \infty)^{2}$. Then the following statements are equivalent:

(i) There exists $\varphi \in \Phi$ such that for any $(t, u) \in D, u \leq \varphi(t)$.

(ii) There exist a nondecreasing, continuous function $\theta$ from $(0, \infty)$ into $\mathbb{R}$ and a function $\psi$ from $\theta((0, \infty))$ into $\mathbb{R}$ satisfying $(\mathrm{U})_{\psi}$ such that for any $(t, u) \in D$, $\theta(u) \leq \psi \circ \theta(t)$.

(iii) There exist a nondecreasing, continuous function $\theta$ from $(0, \infty)$ into $\mathbb{R}$ and an upper semicontinuous function $\psi$ from $\theta((0, \infty))$ into $\mathbb{R}$ satisfying $\psi(t)<t$ such that for any $(t, u) \in D, \theta(u) \leq \psi \circ \theta(t)$.

\section{Competing interests}

The author declares that he has no competing interests.

\section{Acknowledgements}

The author is grateful to the referees for many suggestions to improve the exposition of the paper. The author is supported in part by JSPS KAKENHI Grant Number 25400141 from Japan Society for the Promotion of Science.

Received: 2 December 2015 Accepted: 30 March 2016 Published online: 06 April 2016

References

1. Banach, S: Sur les opérations dans les ensembles abstraits et leur application aux équations intégrales. Fundam. Math. 3, 133-181 (1922)

2. Caccioppoli, R: Un teorema generale sull'esistenza di elementi uniti in una transformazione funzionale. Rend. Accad. Naz. Lincei 11, 794-799 (1930)

3. Browder, FE: On the convergence of successive approximations for nonlinear functional equations. Proc. K. Ned. Akad. Wet., Ser. A, Indag. Math. 30, 27-35 (1968)

4. Ćirić, LjB: A new fixed-point theorem for contractive mappings. Publ. Inst. Math. (Belgr.) 30, $25-27$ (1981)

5. Jachymski, J: Equivalent conditions and the Meir-Keeler type theorems. J. Math. Anal. Appl. 194, $293-303$ (1995)

6. Matkowski, J: Fixed point theorems for contractive mappings in metric spaces. Čas. Pěst. Mat. 105, 341-344 (1980) 
7. Jachymski, J: Around Browder's fixed point theorem for contractions. J. Fixed Point Theory Appl. 5, 47-61 (2009)

8. Jachymski, J: Remarks on contractive conditions of integral type. Nonlinear Anal. 71, 1073-1081 (2009)

9. Kirk, WA: Contraction mappings and extensions. In: Kirk, WA, Sims, B (eds.) Handbook of Metric Fixed Point Theory, pp. 1-34. Kluwer Academic, Dordrecht (2001)

10. Branciari, A: A fixed point theorem for mappings satisfying a general contractive condition of integral type. Int. J. Math. Math. Sci. 29, 531-536 (2002)

11. Suzuki, T: Meir-Keeler contractions of integral type are still Meir-Keeler contractions. Int. J. Math. Math. Sci. 2007, Article ID 39281 (2007)

12. Suzuki, T, Vetro, C: Three existence theorems for weak contractions of Matkowski type. Int. J. Math. Stat. 6, 110-120 (2010)

13. Jleli, M, Samet, S: A new generalization of the Banach contraction principle. J. Inequal. Appl. 2014, Article ID 38 (2014)

14. Branciari, A: A fixed point theorem of Banach-Caccioppoli type on a class of generalized metric spaces. Publ. Math. (Debr.) 57, 31-37 (2000)

15. Alamri, B, Suzuki, T, Khan, LA: Caristi's fixed point theorem and Subrahmanyam's fixed point theorem in $v$-generalized metric spaces. J. Funct. Spaces 2015, Article ID 709391 (2015)

16. Suzuki, T: Generalized metric spaces do not have the compatible topology. Abstr. Appl. Anal. 2014, Article ID 458098 (2014)

17. Suzuki, T, Alamri, B, Khan, LA: Some notes on fixed point theorems in $v$-generalized metric spaces. Bull. Kyushu Inst. Technol., Pure Appl. Math. 62, 15-23 (2015)

18. Suzuki, T, Alamri, B, Kikkawa, M: Only 3-generalized metric spaces have a compatible symmetric topology. Open Math. 13, 510-517 (2015)

19. Suzuki, T: Some notes on Meir-Keeler contractions and L-functions. Bull. Kyushu Inst. Technol., Pure Appl. Math. 53, $1-13(2006)$

\section{Submit your manuscript to a SpringerOpen ${ }^{\circ}$ journal and benefit from:}

- Convenient online submission

- Rigorous peer review

- Immediate publication on acceptance

- Open access: articles freely available online

- High visibility within the field

Retaining the copyright to your article 